\title{
An Experiment in Autonomous Navigation of an Underground Mining Vehicle
}

\author{
Steven Scheding, Gamini Dissanayake, Eduardo Mario Nebot, and Hugh Durrant-Whyte
}

\begin{abstract}
This paper describes the theoretical development and experimental evaluation of a navigation system for an autonomous load, haul, and dump truck (LHD) based on the results obtained during extensive in-situ field trials. ${ }^{1}$ The particular contributions of the theoretical work are in designing the navigation system to be able to cope with vehicle slip in rough uneven terrain using information from inertial sensors, odometry, and a bearing only laser. Results are presented using data obtained during the field trials.
\end{abstract}

Index Terms - Automation, inertial aiding, Kal-man filter, LHD, mining.

\section{INTRODUCTION}

$\mathbf{T}$ The autonomous load, haul, and dump truck (LHD) (see Fig. 1) is the workhorse of the underground mining industry. A typical use for the LHD is in moving ore from the rock face to a centralized dumping point. There is a strong case for automation of these vehicles, for reasons of both safety and productivity. The safety issue has been addressed with several working implementations of teleoperated systems, including the well publicized Canadian systems [1], [2]. Teleoperation, however, offers little to increase productivity, as these systems tend to run at speeds slower than conventional manned systems, resulting in significantly lesser productivity levels with the additional overhead of the infrastructure required to teleoperate. The next step to increasing productivity whilst maintaining safety is therefore to fully automate the LHD, using as little in-mine infrastructure as possible.

Several automatic LHD systems have been tested, including [3] which uses a retroreflective stripe on the tunnel roof (back) detected by cameras to guide the vehicle. This approach, however, requires relatively major levels of infrastructure, which must be changed whenever a new path is required for the vehicle. Similarly, [4] also describes a system that follows an optical path. These approaches may be thought of simply as line following techniques, however the line is detected optically rather than inductively as with buried wire systems [5].

Manuscript received February 5, 1997; revised July 9, 1998. This paper was recommended for publication by Associate Editor R. Chatila and Editor V. Lumelsky upon evaluation of the reviewers' comments.

S. Scheding is with the National Robotics Engineering Consortium, Pittsburgh, PA 15201 USA (e-mail: scheding@ rec.ri.cmu.edu).

G. Dissanayake, E. M. Nebot, and H. Durrant-Whyte are with The University of Sydney, New South Wales 2006, Australia.

Publisher Item Identifier S 1042-296X(99)00818-6.

${ }^{1}$ The experimental work described in this paper was carried out in conjunction with CMTE (CRC for Mining Technology and Equipment) and MIM (Mount Isa Mines Ltd.) and took place at the Mount Isa Lead Mine.
In [6], a system is proposed that uses ultrasonic sensors to follow the walls of a tunnel. The wall following approach suffers from lack of an Earth-fixed coordinate system, which is necessary for any planning operations, particularly traffic management. It is also difficult with wall following to accurately differentiate a side tunnel from a corner.

A more robust approach is presented in [7], where information from various sensors is fused via an Extended Kalman Filter (EKF), to provide an estimate of position and orientation. This approach, however, suffers from under utilization of data. The vehicle model presented uses heading provided from a gyro, coupled with encoder derived velocity information. Information about articulation angle of the vehicle is simply ignored.

A good review of mine automation techniques may be found in $[8]$.

A further obstacle encountered when designing a practical navigation system for underground mining vehicles is the lack of useful data regarding how various sensors perform in an underground environment. It is extremely rare in the literature to find any kind of performance index for the sensors used.

This paper presents data obtained during field trials at an operational underground mine conducted to determine which sensors or combinations of sensors offer a solution to providing a robust navigation system for underground mining vehicles. From these results, a guidance system for an autonomous LHD mining vehicle was designed and is presented here. The key theoretical issues addressed in the design are the explicit modeling of slip parameters as the vehicle maneuvers over rough terrain, and the use of inertial measurements to estimate these parameters.

This paper is broken into two main parts. Section II is a description of the field trials including the experimental setup. Section III details the evolution of, and reasons for a vehicle model which explicitly incorporates slip. This section also details the way the various errors in the system are modeled and the way the sensors themselves are modeled. A results section follows (Section IV) which discusses the results obtained during the field trials as well as an example of the navigation system using data obtained during the field trials, and concluding remarks are made in the final section (Section V).

\section{The Trials Programme}

The automation of underground mining vehicles is not a simple problem. Whilst a relatively large body of information is available for the designer of indoor navigation systems, very little is known about how sensors, and therefore navigation 


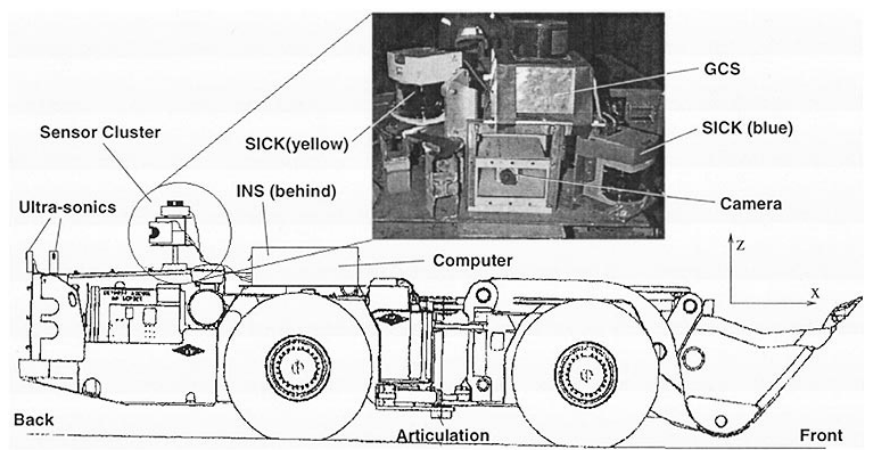

Fig. 1. The LHD—showing sensor layout.

systems, behave in unstructured and harsh environments such as those found in underground mining. To this end, a set of commonly available sensors was tested underground on an LHD mining vehicle over a period of two weeks.

The sensors used in the trials were

1) strap-down inertial navigation system (INS);

2) encoders/odometers;

3) bearing only laser scanner (GCS);

4) two time-of-flight range and bearing laser scanners (SICK);

5) two sets of ultrasonic sensors (MASA/Polaroid).

These sensors may be broken down into two broad categories, dead reckoning sensors, and external sensors. While dead reckoning sensors tend to be very robust, they accumulate error with time, so that in practise they must be periodically reset using information from the external sensors. The external sensors provide absolute information referenced to the environment, typically by making measurements of prominent known landmarks, whether they be natural or artificial. The external sensors, however, do not tend to be as reliable as dead reckoning sensors, so a navigation package should use sensors of both types.

Fig. 1 shows an LHD and the sensor layout used in the underground trials ${ }^{2}$

\section{A. Experimental Setup}

The data from the different sensors needed to be recorded so that it could be post-processed. To achieve this, a distributed network of computers was located on an LHD, synchronized by a time stamp sent from the master computer to the slaves at a rate of $50 \mathrm{~Hz}$. This enabled the data from each sensor to be individually time stamped. This is important as some of the sensors produce data asynchronously. The overall system is shown in Fig. 2.

Fig. 3 shows the area used during the trials. The tunnel is approximately 150 meters long. The sensor data was collected while the LHD was running back and forward between the black crosses marked 1 and 2 . The small square in the figure shows the area of the tunnel for which results will be presented.

\section{B. Suggested Navigation Systems}

From the results of the field trials, two main navigation solutions have presented themselves which are constrained

\footnotetext{
${ }^{2}$ Figs. 1 and 2 were created by J. Roberts of CMTE.
}

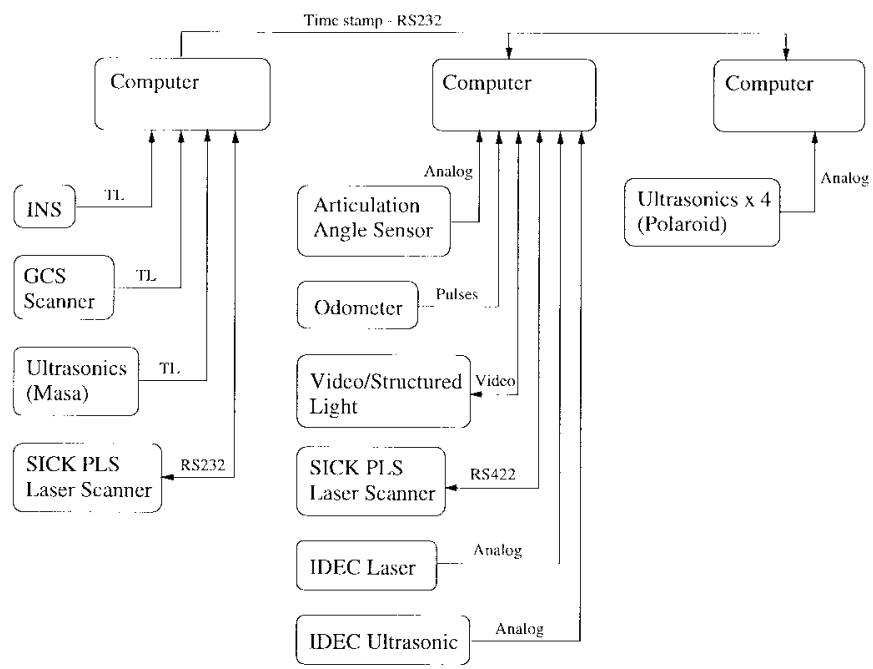

Fig. 2. The data logging system.

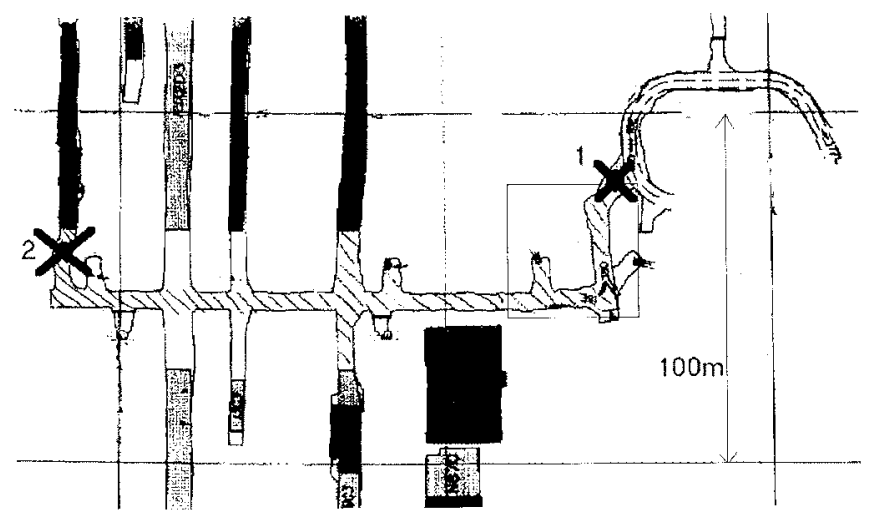

Fig. 3. The trials area.

by the wish for low infrastructure. The first is a geometric solution in which encoder based dead reckoning is fused with periodic updates to artificial landmarks using a sensor such as the GCS laser. This solution requires infrastructure, though far less than existing systems. The second solution is one that is feature based. INS based dead reckoning is fused with sensor information of natural landmarks, such as the SICK laser or ultrasonics. Ideally, the two navigation systems will operate in parallel, providing robustness through redundancy. The inherent robustness is further enhanced through the use of different sensing technologies for each navigation system, as each separate sensor will have unique failure modes. In systems which use double or triple redundancy of a single sensor, the failure modes of the sensors may be identical and therefore fail simultaneously.

The feature based navigation system is still a research issue at the preliminary stages of investigation, so only results from the geometric navigation system will be presented in this paper.

\section{The Geometric Navigation System}

This section of this paper is devoted to the detailed derivation and implementation of the first suggested navigation solution, that of encoder based dead reckoning together with 


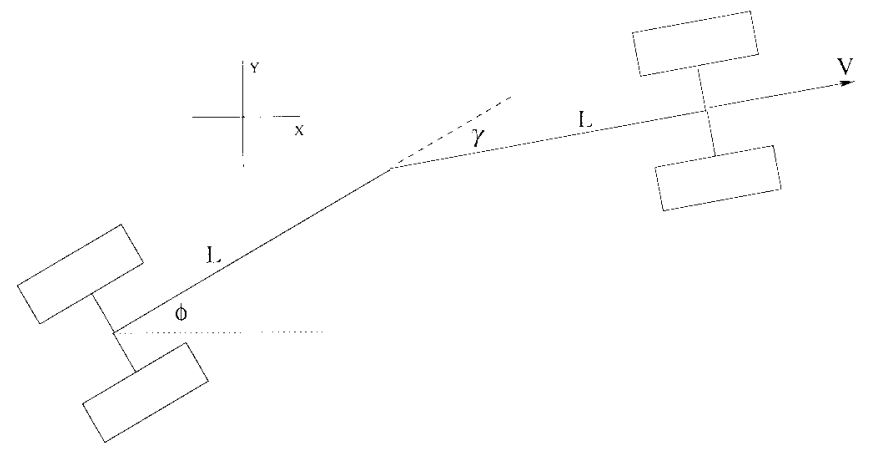

Fig. 4. LHD kinematic geometry.

the GCS laser. A gyro is also used to aid in the estimation of vehicle slip, which was found to be a nontrivial problem. All data used in the following sections was obtained during the field trials.

\section{A. The Vehicle Model}

The key to designing navigation systems for autonomous vehicles is the model which describes how the vehicle's position and other key vehicle parameters evolve through time. The model is essential to make good use of sensor data and for describing how unobserved vehicle parameters affect vehicle motion. This model usually consists of two parts, the vehicle model itself, and a model which describes how errors in the vehicle model propagate in time.

The LHD is an articulated vehicle, which means that the vehicle has a front and rear body which can rotate relative to each other. The front and rear wheel sets are fixed to remain parallel with the body of the vehicle, and steering is achieved by driving the articulation joint located midway between the front and rear axles. Fig. 4 shows the geometry of a typical LHD.

1) The No-Slip Model: The kinematic model of the vehicle derived from rigid body and rolling motion constraints is given by

$$
\begin{aligned}
\dot{x}(t) & =V \cos (\phi) \\
\dot{y}(t) & =V \sin (\phi) \\
\dot{\phi}(t) & =\frac{V \tan \left(\frac{\gamma}{2}\right)}{L}
\end{aligned}
$$

where $x$ and $y$ denote the position of the vehicle relative to some fixed global frame of reference. The angle $\phi$ is the orientation of the vehicle with respect to the $x$ axis, while $V$ represents the linear velocity of an imaginary front wheel located midway between the real front wheels. The angle $\gamma$ is defined as the articulation angle of the vehicle, and $L$ is the half-length of the vehicle, the distance between the front or rear set of wheels and the articulation joint. These equations are based on the assumption that the front and rear wheel velocities of the LHD are identical and $\gamma$ is a constant. The derivation is omitted here, but the result concurs with [6].

However, in practice the articulation angle changes rapidly and in fact is the means used for steering the vehicle. Slip must therefore occur due to the overdetermination of the wheels' speeds. This means that the constraint of zero velocity in the

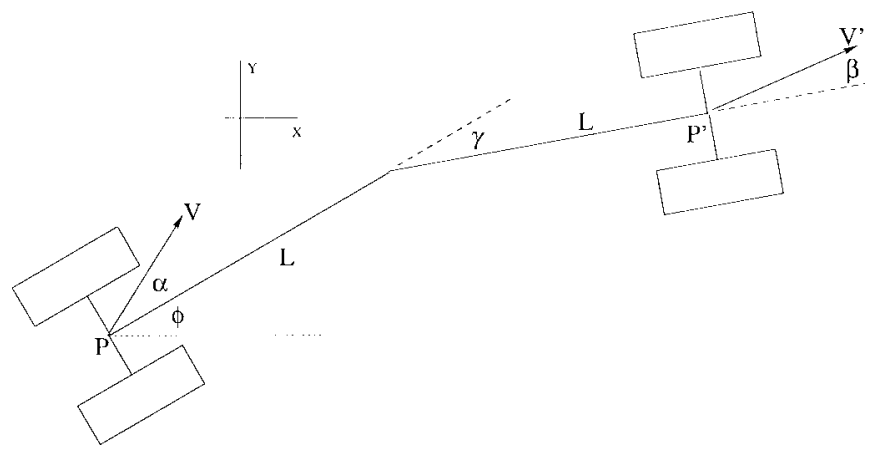

Fig. 5. LHD kinematic geometry—including slip angles.

direction of the axles (rolling motion constraint) is not valid, and in fact this model greatly overestimates the rate of change of orientation $\dot{\phi}$. A new kinematic model that explicitly models this slip is therefore developed as follows.

2) Accounting for Slip: To take into account that the vehicle will slip during motion, two slip variables $\alpha$ and $\beta$ are introduced. These variables are chosen to represent the slip angles of the vehicle, that is, the angle between the kinematically indicated velocity (perpendicular to the axles) and the true velocity. The variation between true and kinematic velocities is by definition entirely dependent on the slip.

Consider the velocity of the point $P$ in the rear body, and the velocity at a symmetric point $P^{\prime}$ in the front body as shown in Fig. 5. Generally, the prime will indicate quantities related to the front of the vehicle, whilst no prime indicates quantities related to the rear.

In this paper, all quantities are referenced to the rear of the vehicle, as this is where the sensor array was located. As will be seen, the articulation angle is considered an uncertain parameter, making coordinate transforms from the front to rear of the vehicle a nontrivial problem.

By obtaining the velocity of $P^{\prime}$ in the direction perpendicular to $V^{\prime}$ and equating this to zero, we obtain the following relation:

$$
V \sin (\alpha-\beta-\gamma)+L \cos (\beta+\gamma) \dot{\phi}+L \cos (\beta)(\dot{\phi}+\dot{\gamma})=0
$$

which may be solved for $\dot{\phi}$ to yield

$$
\dot{\phi}=\frac{(\omega R \sin (\beta-\alpha+\gamma)-\dot{\gamma} L \cos (\beta))}{L(\cos (\beta)+\cos (\beta+\gamma))} .
$$

If the velocity $V$ of $P$ is now set to equal $\omega R$, the wheels angular velocity multiplied by the nominal wheel radius, and resolved into $x$ and $y$, the following kinematic equations for the motion of point $P$ are obtained:

$$
\begin{aligned}
& \dot{x}=\omega R \cos (\alpha+\phi) \\
& \dot{y}=\omega R \sin (\alpha+\phi) \\
& \dot{\phi}=\frac{(\omega R \sin (\beta-\alpha+\gamma)-\dot{\gamma} L \cos (\beta))}{L(\cos (\beta)+\cos (\beta+\gamma))} .
\end{aligned}
$$

It can be seen that the vehicle moves in the direction given by the slip angle, i.e., the vehicle heads in the direction $\alpha+\phi$, whilst the rate of change of orientation is dependant on the slip angles, the articulation angle, and the rate of change of articulation angle. 
3) The Importance of Slip: Comparing (2) to (1), it can be seen that the models are significantly different, particularly for the term $\dot{\phi}$. The simple model described by (1) greatly overestimates the turning rate of the vehicle, causing the navigation system to continuously fight to correct the modeling error. Equation (2) is far more accurate if the slip angles are known. This is where the Extended Kalman Filter (EKF) plays an important role, because states that are not directly observable, such as the slip parameters, may be estimated and used to improve the model of the vehicle.

As the vehicle drives both the front and rear wheel sets at the same angular velocities, insight into why the vehicle does indeed slip may be gained by examining the different expressions for $V$ and $V^{\prime}$. The equation governing $V^{\prime}$ is

$$
V^{\prime}=\frac{V(\cos (\alpha-\gamma)+\cos (\alpha))-L \dot{\gamma} \sin (\gamma)}{\cos (\beta)+\cos (\beta+\gamma)}
$$

When the slip angles $\alpha$ and $\beta$ are zero (i.e., no slip is present), we obtain the following expression for the velocity of the front wheel:

$$
V^{\prime}=V-L \dot{\gamma} \tan \left(\frac{\gamma}{2}\right)
$$

It is clear from this that the only conditions under which the front and rear velocities are equal (under no-slip assumptions) is when the articulation angle $\gamma$ is constant or zero. Therefore wheel slip must be present. Experimental data shows that the rate of change of articulation angle $\dot{\gamma}$ can be quite high, and may cause the vehicles tyres to scrub the ground. Human operators tend to use this effect to their advantage.

4) The Error Model: When the model addressed in (2) is considered, it can be seen that the primary sources of error are due to the time varying parameters $\omega, \gamma, \dot{\gamma}, \alpha$ and $\beta$, as errors in these parameters propagate directly through to the states. The variables $\omega, \gamma$ and $\dot{\gamma}$ represent well known control inputs and thus do not need to be estimated. The slip parameters $\alpha$ and $\beta$, however, are not directly measured, and therefore cannot be treated in the same way as the control inputs. It is interesting to note that the effective wheel radius of the vehicle can also be considered to be time varying and takes into account factors such as loading and tyre wear. The wear on a typical LHD tyre can be as much as $15-20 \mathrm{~cm}$ in radius and will therefore introduce a bias to the system over the operating lifetime of the tyres if a constant wheel radius is assumed. The concept of estimating wheel radius was introduced in [9], and is extremely beneficial to this application. Therefore, the states to be estimated should not only include position and orientation, but also the slip angles and wheel radius.

It is salient at this point to describe how the error in each of the parameters may be modeled, as it is important to know how the errors propagate through time. The errors in control input are modeled as simple additive noise $\delta \omega(t), \delta \gamma(t)$ and $\delta \dot{\gamma}(t)$ about their respective means $\bar{\omega}(t), \bar{\gamma}(t)$ and $\dot{\bar{\gamma}}(t)$ at time $t$ such that

$$
\begin{aligned}
\omega(t) & =\bar{\omega}(t)+\delta \omega(t) \\
\gamma(t) & =\bar{\gamma}(t)+\delta \gamma(t) \\
\dot{\gamma}(t) & =\dot{\bar{\gamma}}(t)+\delta \dot{\gamma}(t)
\end{aligned}
$$

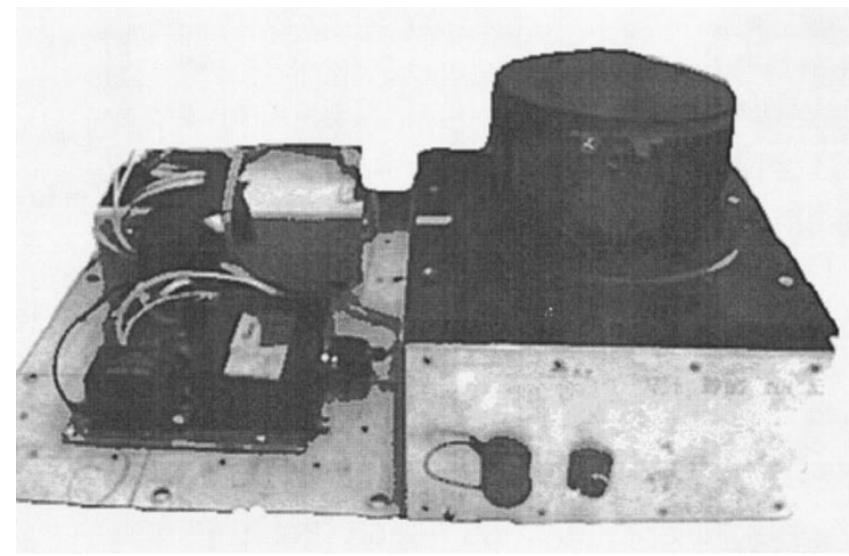

Fig. 6. The INS and laser used in the field trials.

The errors in $R, \alpha$ and $\beta$, however, are extremely difficult to model accurately as they tend to involve a combination of other parameters, and are caused fundamentally by the vehicle dynamics. For example, the error in slip angle will change with respect to vehicle speed, mass, tyre-terrain interaction, and articulation angle in a highly nonlinear way. A compromise which has been found to work well is to model the errors in these parameters as random walks (or Brownian motion), such that the error in each of the parameters is the integral of white noise as follows:

$$
\begin{aligned}
\dot{R}(t) & =\delta R(t) \\
\dot{\alpha}(t) & =\delta \alpha(t) \\
\dot{\beta}(t) & =\delta \beta(t) .
\end{aligned}
$$

The noise sources $\delta \omega(t), \quad \delta \gamma(t), \quad \delta \dot{\gamma}(t), \quad \delta R(t), \quad \delta \alpha(t)$ and $\delta \beta(t)$ are assumed zero-mean, uncorrelated, gaussian sequences with variance $\sigma_{\omega}^{2}, \sigma_{\gamma}^{2}, \sigma_{\dot{\gamma}}^{2}, \sigma_{R}^{2}, \sigma_{\alpha}^{2}$ and $\sigma_{\beta}^{2}$, respectively. Although in practice these parameters may not evolve in a strictly Brownian manner, the Brownian model reflects the growth in uncertainty in their true value, and the rate at which the true value is considered to vary.

The continuous time vehicle model shown in (2) may now be rewritten at time $t$, adding the additional states to be estimated as

$$
\begin{aligned}
\dot{x}(t)= & \omega(t) R(t) \cos (\alpha(t)+\phi(t)) \\
\dot{y}(t)= & \omega(t) R(t) \sin (\alpha(t)+\phi(t)) \\
\dot{\phi}(t)= & \frac{\omega(t) R(t) \sin (\beta(t)-\alpha(t)+\gamma(t))}{L(\cos (\beta(t))+\cos (\beta(t)+\gamma(t)))} \\
& -\frac{\dot{\gamma}(t) L \cos (\beta(t)))}{L(\cos (\beta(t))+\cos (\beta(t)+\gamma(t))} \\
\dot{R}(t)= & \delta R(t) \\
\dot{\alpha}(t)= & \delta \alpha(t) \\
\dot{\beta}(t)= & \delta \beta(t) .
\end{aligned}
$$

5) Observation Model: The vehicle has a GCS laser sensor (see Fig. 6) which is capable of detecting the angle to a number of fixed beacons $\mathbf{B}_{i}=\left[X_{i}, Y_{i}\right]^{T}, i=1, \cdots, N$. This sensor, although in practise very good, only allows the state to be updated periodically, or at a low frequency, due to its $1 \mathrm{~Hz}$ rotation rate. The laser observations are compared to a map 

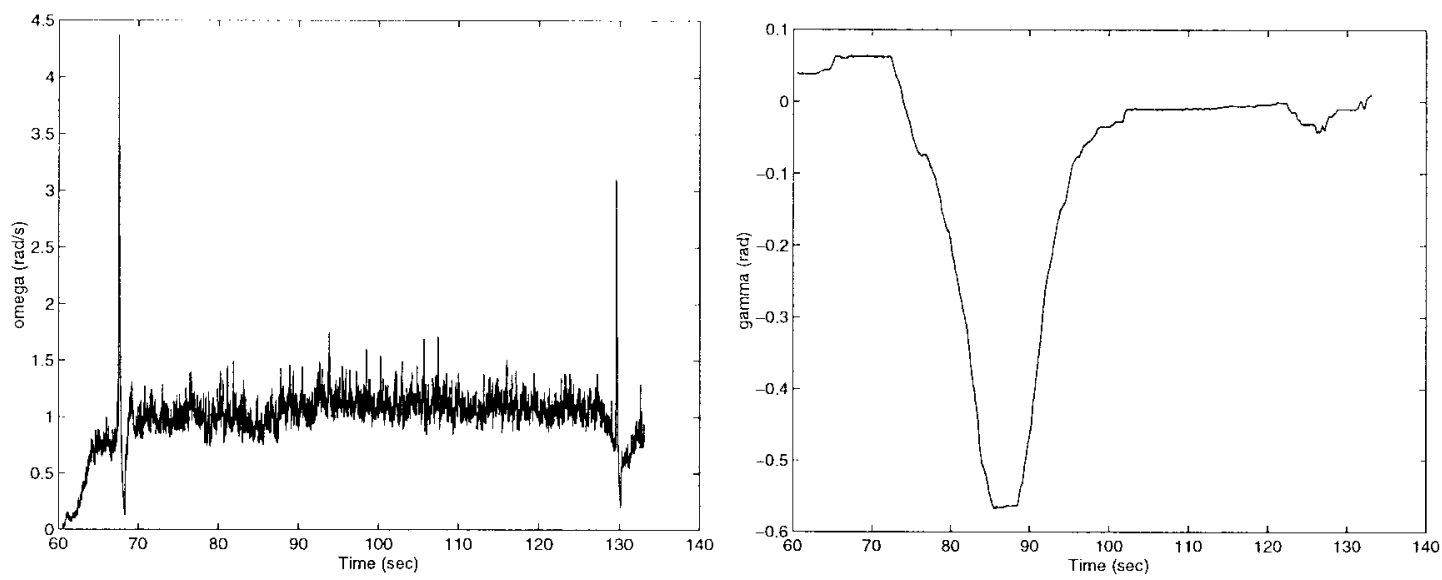

Fig. 7. Vehicle velocity and steer angle.

of known beacon locations, and are validated statistically to determine the correct match.

To augment the system with gyro information, information from the three gyros is first converted to a single rotation about the vehicles $z$ axis, then fused in the filter to provide a better estimate of state. Gyros are well known to drift with time, a low frequency error which is fed straight through to the estimate if the gyro drift is not compensated for. By using a shaping filter however, the low frequency errors in the gyro information may be modeled, and are thus estimated by the filter, so as not to corrupt the vehicle pose estimate.

The errors in the gyro are adequately modeled as Brownian motion, the integral of white noise. The shaping state for the gyro error $x_{s f}$ is therefore given by

$$
\dot{x}_{s f}(t)=\mathbf{w}_{s f}(t) \text {. }
$$

Where $\mathbf{w}_{s f}(t)$ represents a gaussian sequence with variance $\sigma_{s f}^{2}$. The shaping state must now be used to augment the state vector as follows:

$$
\mathbf{x}(t)=\left[x(t), y(t), \phi(t), R(t), \alpha(t), \beta(t), x_{s f}(t)\right]^{T} .
$$

The observations which the sensors will make are assumed to be of the form

$$
\mathbf{z}(t)=\mathbf{h}(\mathbf{x}(t))+\mathbf{w}(t)
$$

where $\mathbf{z}(t)$ represents the observation vector, $\mathbf{h}(\cdot)$ is the nonlinear matrix mapping observations to states and $\mathbf{w}(t)$ is additive sensor noise, assumed gaussian and with zero mean.

The bearing to a beacon may be given by $\arctan \left(\frac{Y_{i}-y(t)}{X_{i}-x(t)}\right)$, however the vehicle is oriented in the direction $\phi$, so for this system, the measurement equation for each beacon detected by the laser is given by the nonlinear model

$$
\mathbf{z}_{\theta}^{i}(t)=\left[\arctan \left(\frac{Y_{i}-y(t)}{X_{i}-x(t)}\right)-\phi(t)\right]+\left[w_{\theta}^{i}(t)\right]
$$

and the measurement equation for the gyro is given by the linear model

$$
\mathbf{z}_{\mathrm{gyro}}(t)=\left[\phi(t)+x_{s f}(t)\right]+\left[w_{\mathrm{gyro}}(t)\right]
$$

which is an observation of the orientation plus the shaping state representing gyro drift.

The beacon locations were surveyed and therefore presumed precisely known. To validate this data to avoid the incorporation of spurious measurements, a gate function based on a $\chi^{2}$ test was used. This is explained in more detail in [9].

\section{B. Implementing the Navigation System}

To implement a Kalman Filter based on a continuous time model, it is convenient to discretely the model, as the control signals $\omega$ and $\gamma$ and the sensor observations are sampled at regular discrete intervals.

The discrete EKF algorithm [10] consists of a predictionupdate cycle where prediction is performed when either no sensor information is available or to predict the state at the next discrete time-step. An update occurs when sensor information is available to improve the systems estimate of state. Similar data fusion issues are addressed in [9], [11], and [12]. The filter derivation for this system is found in the Appendix.

\section{RESULTS}

\section{A. The Trials}

The following sections summarize the results obtained from the sensors used in the underground trials. Particular attention is paid to those sensors used in the navigation filter. That is, the odometry sensors, the GCS laser, and the gyros contained within the INS.

1) Odometry: In this work, an existing inductive sensor was used to measure the angular velocity of the LHD's transmission, while a potentiometer was used to measure the articulation angle. Fig. 7 shows velocity and articulation angle of the vehicle during a 70-s period of the run. The spikes in the velocity estimate are due to inadequate hardware, however the mean of the velocity is still approximately correct, as required by the Kalman filter derivation.

Typically with odometry based dead reckoning, information from wheel rate sensors and steer angle sensors is passed through the particular vehicles forward kinematic equations to obtain an estimate of position and orientation. A problem with this form of dead reckoning is that the vehicle model is 


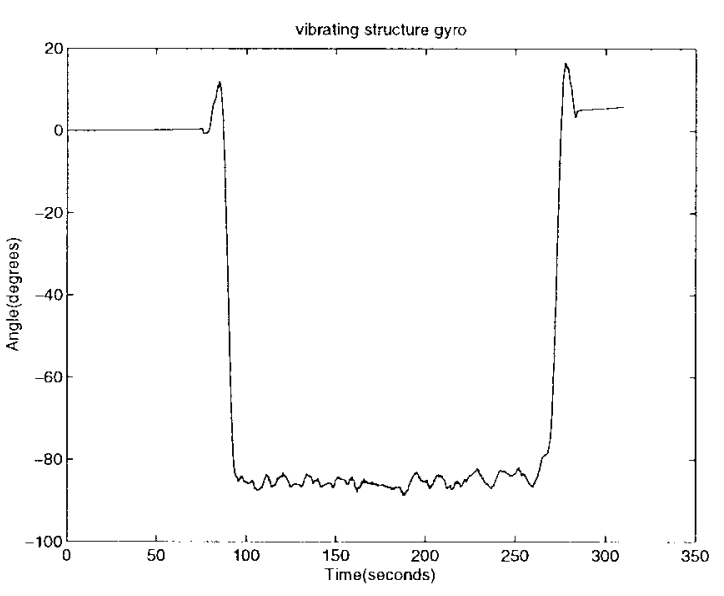

(a)

Fig. 8. Yaw: (a) vibrating structure and (b) fiber optic.

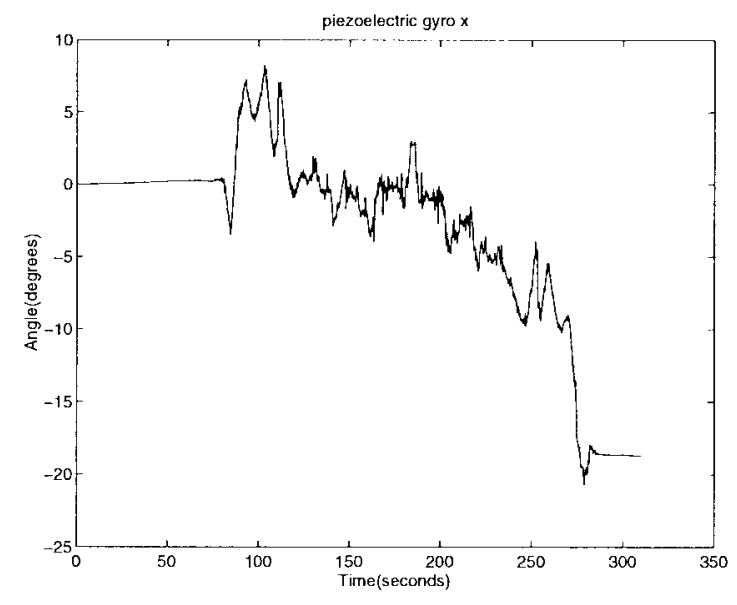

Fig. 9. Piezoelectric gyro $x$ and $y$.

usually derived in two dimensions, so the further the terrain is from a plane, the less accurate the solution. Another problem is that odometry based dead reckoning cannot identify wheel slip. Odometry remains, though, as the cheapest way of obtaining dead reckoning information reliably.

Encoders were not used in this application as they are generally regarded as insufficiently robust in harsh environments.

2) Inertial Sensors: The INS system used in the trials programme [13] is a strap-down system consisting of a single triaxial accelerometer and four gyros. The accelerometer provides three analog outputs proportional to the acceleration on each of three orthogonal axes. Two gyros were used for measuring roll and pitch rates. Two different gyros were used for measuring the rotation rate of the vehicle around vertical yaw. These two gyros are significantly more accurate and expensive than the roll and pitch gyros. The gyros were distributed in this form because in many applications yaw (heading) will be the only inertial information required. Two solid state gyros were used for the Roll $(y)$ and Pitch $(x)$ axes. These sensors employ a vibrating piezoelectric prism which distorts and produces a voltage due to the Coriolis force generated by angular velocity. The first heading gyro is a single axis interferometric fiber optic gyroscope. The second

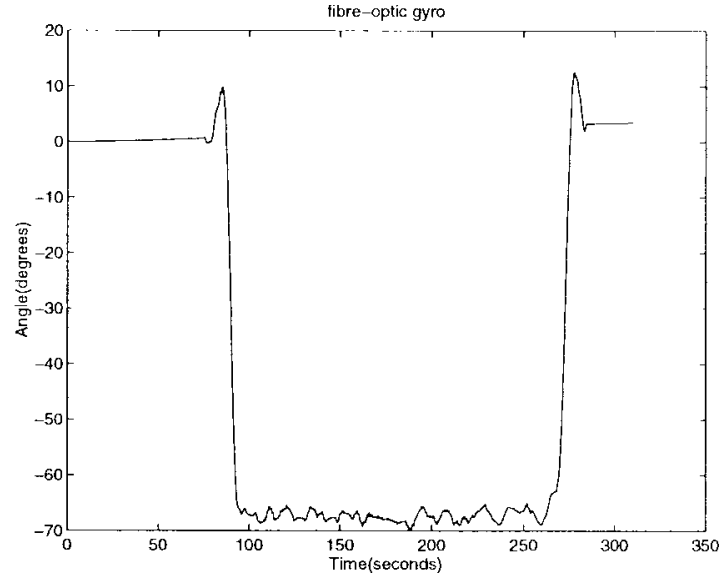

(b)

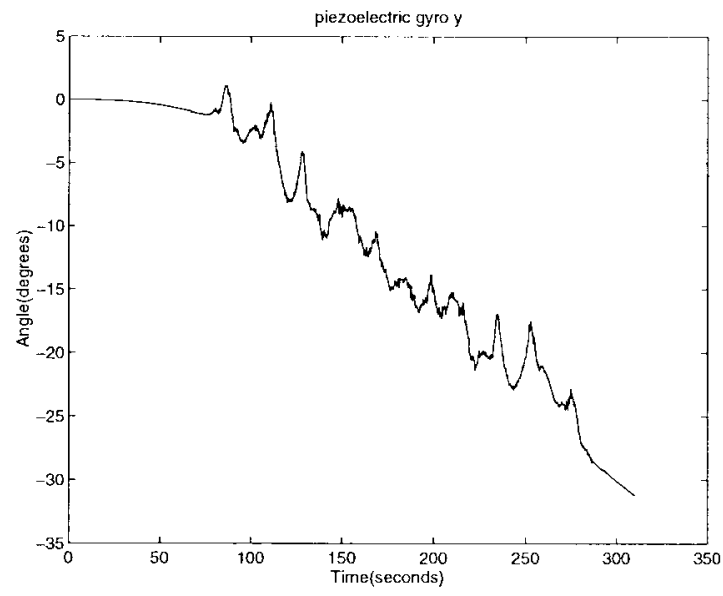

heading gyro is a vibrating structure type Gyro with very low drift characteristics.

Gyros. The two best gyroscopes were used to record heading (yaw) data. Typical drift rates for these devices were in the order of $0.2 \% \mathrm{~min}$. Non-linearities in the gyros result in errors in the measured rotations. This is especially prominent when the gyro is rotated and returned to the zero position. Typical accumulated errors for a 90 excursion are in the order of 1 degree for these type of gyros.

Typical heading results for the gyros are shown in Fig. 8. The figure shows the vehicle starting out at zero degrees, turning a near ninety degree corner, stopping, reversing and turning back round the corner to the starting point. The gyros were mounted on the rear of the LHD articulation. As the LHD starts to turn, first corner in Fig. 8, the rear articulation initially turns in the opposite direction before beginning the main turning operation (the front of the articulation will equally over-steer when coming out of the corner). This is clearly seen in the data at the start of the turn and at the end of the return corner.

From these plots it can be seen that these gyros provide angular information that can be used for long period of time without any on line calibration. Fig. 9 shows typical roll 


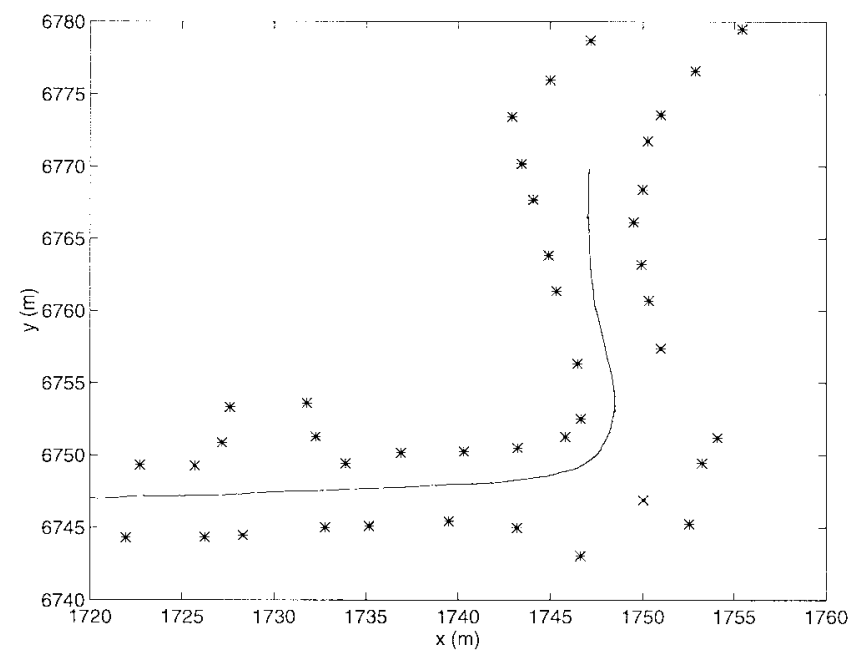

Fig. 10. Estimated LHD path—stars represent retroreflective markers.

and pitch angles recorded by integrating the output of the piezoelectric gyros.

These gyros are particularly sensitive to large zero offset errors. Static drift rates at a stable temperature are in the order of $5 \% \mathrm{~min}$. In addition, these devices also suffer from some hysteresis with the zero offset varying to a new random value after motion. The offset varies by over $1 \% \mathrm{~min}$ from this source of error alone. The linearity of these devices also seems to be variable. The attitude of the platform can be obtained integrating the measurements of roll, pitch and yaw angles from gyroscopes. The orientation angles must be estimated very accurately as even a small fraction of gravitational acceleration attributed to true linear acceleration results in huge position errors. The yaw gyros used for measuring angular rates around the vertical axis are sufficiently accurate to obtain yaw information. However, the roll and pitch gyros used in the INS package were not sufficiently accurate to provide sensible estimates of roll and pitch angles even over short periods of time.

On-line calibration of these sensors is possible however, and the interested reader is referred to [14] and [15].

3) GCS Laser: The guidance control systems (GCS) laser is shown in Fig. 6. The laser's functionality is described previously in the Observation Model section of this paper. Fig. 10 shows a portion of the reflective beacons mounted on the tunnel walls.

From the trials, this sensor was found to be extremely reliable. Although the sensor was shock-mounted in order to reduce vibration due to vehicle motion, the only problem found was due to the vibration of the sensor. The problem, encountered only occasionally during high speed runs, is that the sensor appears to detect a single target more than once, since the vibration of the vehicle can induce a net, although very slight, backward motion in the sensor.

\section{B. The Navigation System}

This section presents the results of implementing the navigation system described previously in this paper. The results were obtained using data from the field trials on an LHD in

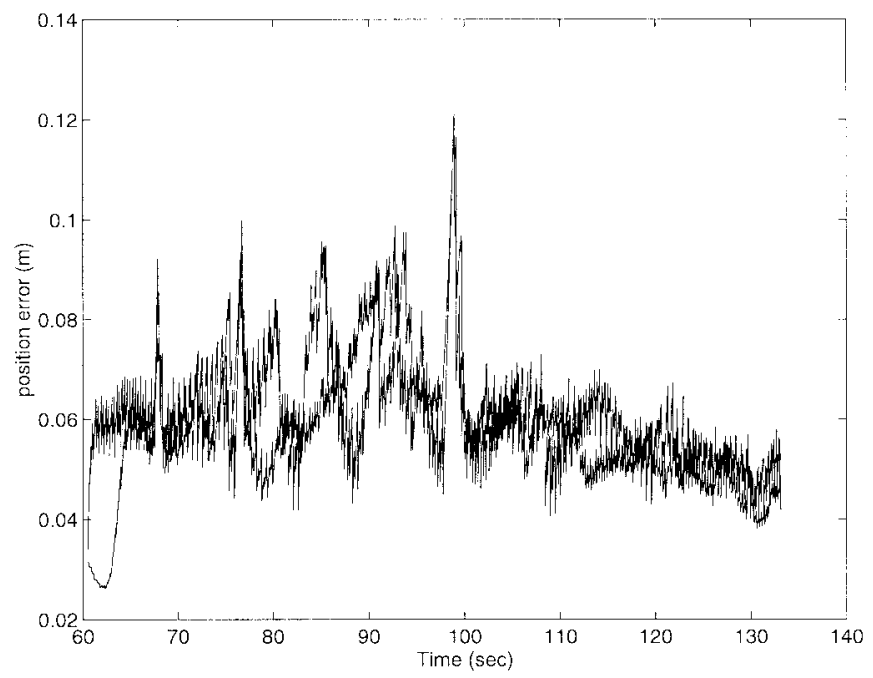

Fig. 11. Position standard deviation during run ( $x$ and $y$ ).

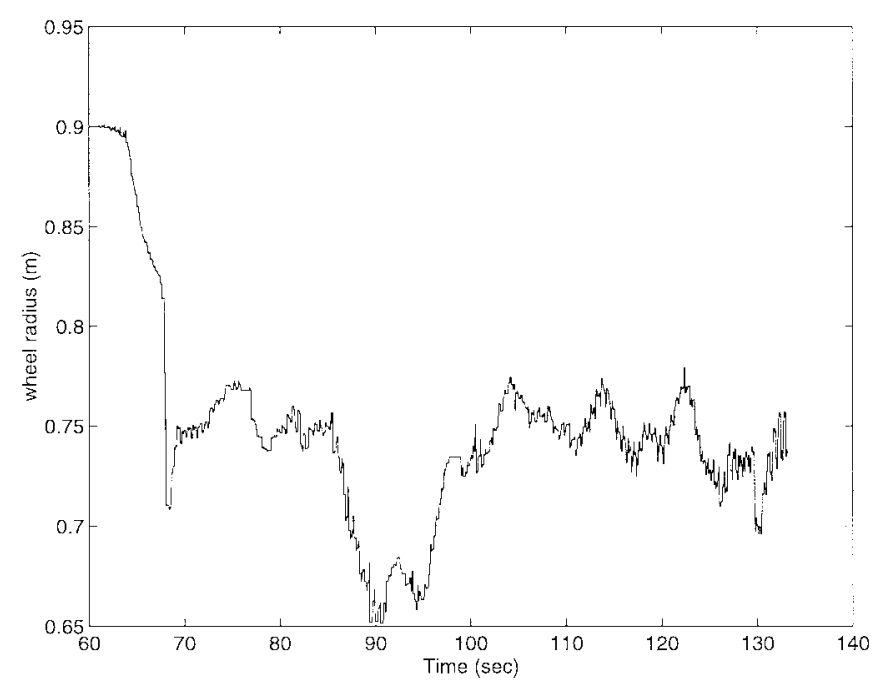

Fig. 12. Estimated wheel radius.

an underground mine in Queensland, Australia. The sensors and their layout is shown in Fig. 1. The sensor positions were measured accurately so that coordinate transformations could be reliably achieved. The experimental setup is described in earlier sections, and also in [16].

The trials themselves utilized a section of tunnel approximately one hundred and fifty meters long, populated with retroreflective strips used as beacons for the laser scanner and is shown in Fig. 3. The strips were surveyed to provide their positions in the tunnel. The data from all sensors on board the vehicle was logged and time-stamped during the trials, and post processed to provide the results seen here.

Fig. 10 shows the tracked path of the LHD during a greater than right angle turning manoeuvre. The maximum standard deviation, or error bound in position during this manoeuvre (as estimated by the EKF) was approximately eight centimeters as can be seen in Fig. 11. The spikes in the standard deviation correspond to periods during the run when no beacons could be seen or accurately identified. Figs. 12-14 show the estimated wheel radius and the slip angles $\alpha$ and $\beta$, respectively. These 


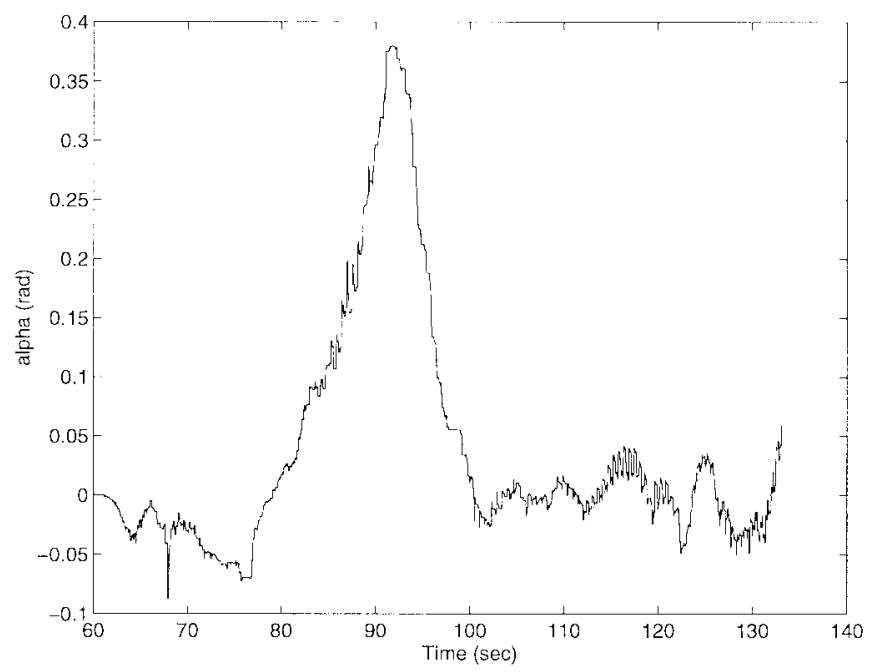

Fig. 13. Estimated slip angle $\alpha$.

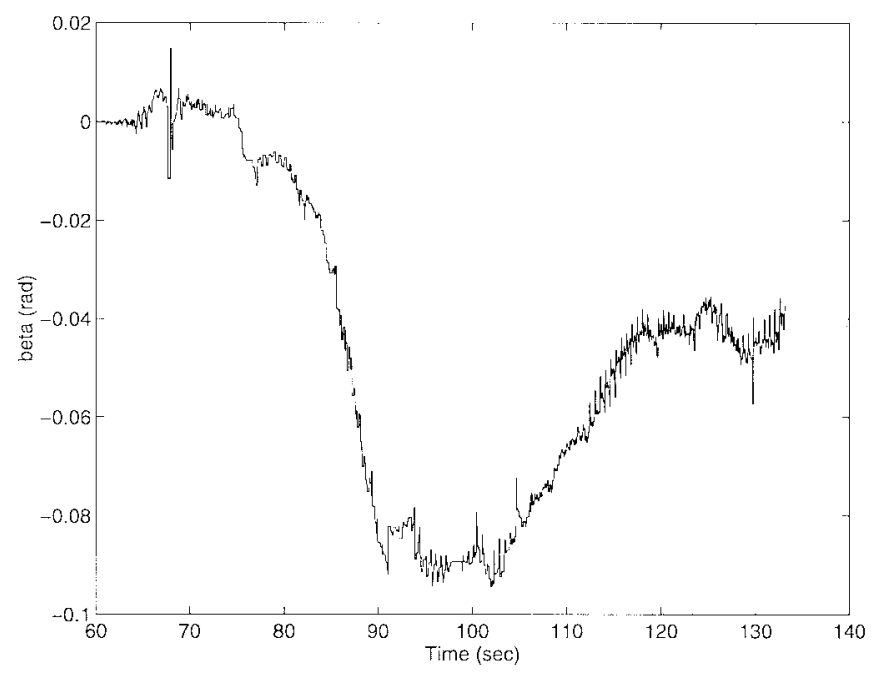

Fig. 14. Estimated slip angle $\beta$.

may be compared to Fig. 7 which shows the variation in articulation angle over time. As can be seen from these graphs, the estimated slip is highly correlated with the articulation angle, verifying the intuitive assumption that the vehicle will slip more while cornering. This is of particular importance in underground mining where a majority of the tunnel corners are tight right angled bends. In this example, the forward slip angle $\beta$ approaches $6^{\circ}$ during the corner, while the rear slip angle $\alpha$ exceeds $20^{\circ}$. From the kinematic model of the vehicle shown in Fig. 5, it can be seen that the sign of the slip angles corresponds to the vehicle turning the corner faster than the no-slip model would allow. The magnitude of the slip angles indicates that the rear of the vehicle slips more than the front. Wheel slip is a function of many factors such as the normal force between the tyre and ground, the tyre stiffness, and mass distribution. The differences in the slip angles observed may be attributed to these effects. Fig. 12 shows that the estimated wheel radius quickly converges from the initial value of $0.9 \mathrm{~m}$ to a value of approximately 0.75 $\mathrm{m}$, which was in fact the correct wheel radius at the time of the trials. It is also interesting to note that the estimated wheel radius $R$ decreases by approximately $0.1 \mathrm{~m}$ during the turning manoeuvre. The reason for this effect is clear when we consider that the vehicles forward velocity is given by $V=\omega R$. Any change in the forward velocity of the vehicle (due to forward slip) is estimated as a change in wheel radius by the EKF. The slip angles account for the across axis, or transverse, slip.

Further experimental results (not shown) based on the data recorded during other runs show that the filter reliably estimates vehicle position over the entire operating range of vehicle speeds. The only noticeable difference between the slow and high speed runs is the estimated position standard deviation, which is larger at higher speeds.

\section{CONCLUSION}

In this paper, the results of a series of tests conducted in an underground mine to determine which sensor or sensor combinations will provide reliable guidance for a mining vehicle was presented. Two suggested navigation systems were the result. From the data, it was concluded that the vehicle slips to a large extent, so an LHD vehicle model which explicitly incorporates slip was developed. A navigation system was described in which observations from a laser sensor and from an INS unit are incorporated to aid the estimation of the slip angles. The results clearly show that the amounts of slip experienced by the vehicle are nonnegligible particularly during cornering. In this case, the no-slip model simply fails. The benefits of this approach are in making the navigation systems for large heavy industrial machinery much more reliable and robust in harsh uneven terrain. Future research in this area will include the addition of a full INS unit, including accelerometers to aid the navigation system, and research into the use of redundant navigation packages to detect system faults. The theory described in this paper will be extended for use in outdoor applications. Further long term trials are needed to determine sensor degradation patterns.

\section{APPENDIX}

\section{A. Discrete Vehicle Model}

The control signals $\omega$ and $\gamma$ and the sensor observations are sampled at regular discrete intervals. The sample interval $\Delta T$, is synchronous and small enough to capture maneuvers occurring in the operating frequency range. The control inputs are assumed approximately constant over this interval. Replacing the continuous time index $t$ with a discrete time index $k$, (3) becomes (9), shown at the bottom of the next page.

The discrete time state vector at time $k+1$ can now be defined as

$$
\begin{aligned}
\mathbf{x}(k+1)= & \mathbf{f}(\mathbf{x}(k), \mathbf{u}(k), k)+\mathbf{v}(k) \\
= & {[x(k+1), y(k+1), \phi(k+1), R(k+1)} \\
& \left.\alpha(k+1), \beta(k+1), x_{s f}(k+1)\right]^{T}
\end{aligned}
$$

where

$$
\mathbf{u}(k)=[\omega(k), \gamma(k), \dot{\gamma}(k)]^{T}
$$


is the control vector at time $k, \mathbf{v}(k)$ is additive process noise representing the uncertainty in slip angles and wheel radius and $\mathbf{f}(\cdot)$ is the nonlinear state transition function mapping the previous state and current control inputs to the current state, here represented by (9).

\section{B. State Prediction}

Assume the system has an estimate available at time $k$ which is equal to the conditional mean

$$
\hat{\mathbf{x}}(k \mid k)=E\left[\mathbf{x}(k) \mid \mathbf{Z}^{k}\right] .
$$

The prediction $\hat{\mathbf{x}}(k+1 \mid k)$ for the state at time $k+1$ based on information up to time $k$ is given by expanding (10) as a Taylor series about the estimate $\hat{\mathrm{x}}(k \mid k)$, eliminating second and higher order terms, and taking expectations conditioned on the first $k$ observations, giving

$$
\hat{\mathbf{x}}(k+1 \mid k)=E\left[x(k+1) \mid \mathbf{Z}^{k}\right]=\mathrm{f}(\mathbf{x}(k \mid k), \mathbf{u}(k), k) .
$$

The vector state prediction function $\mathrm{f}(\cdot)$ is defined by (9) assuming zero process and control noise. The prediction of state is therefore obtained by simply substituting the previous state and current control inputs into the state transition equation with no noise.

Define the noise source vector as

$$
\begin{aligned}
& \delta \mathbf{w}(k) \\
& \quad=\left[\delta \omega(k), \delta \gamma(k), \delta \dot{\gamma}(k), \delta R(k), \delta \alpha(k), \delta \beta(k), \delta \mathbf{w}_{s f}(k)\right]^{T} .
\end{aligned}
$$

The error between the true state and the estimated state is given by

$$
\delta \mathbf{x}(k \mid k)=\mathbf{x}(k)-\hat{\mathbf{x}}(k \mid k)
$$

the prediction of covariance is then obtained as

$$
\begin{aligned}
\mathbf{P}(k+1 \mid k)= & E\left[\delta \mathbf{x}(k \mid k) \delta \mathbf{x}(k \mid k)^{T} \mid \mathbf{z}^{k}\right] \\
= & \nabla \mathbf{f}_{\mathbf{x}}(k) \mathbf{P}(k \mid k) \nabla \mathbf{f}_{\mathbf{x}}^{T}(k) \\
& +\nabla \mathbf{f}_{\mathbf{w}}(k) \boldsymbol{\Sigma}(k) \nabla \mathbf{f}_{\mathbf{w}}^{T}(k)
\end{aligned}
$$

where $\nabla \mathrm{f}_{\mathbf{x}}(k)$ represents the gradient or Jacobian of $\mathbf{f}(\cdot)$ evaluated at time $k$ with respect to the states, $\nabla \mathrm{f}_{\mathbf{w}}(k)$ is the Jacobian of $\mathbf{f}(\cdot)$ with respect to the noise sources, and $\boldsymbol{\Sigma}(k)$ is the noise strength matrix given by

$$
\Sigma(k)=\left[\begin{array}{ccccccc}
\sigma_{\omega}^{2} & 0 & 0 & 0 & 0 & 0 & 0 \\
0 & \sigma_{\gamma}^{2} & 0 & 0 & 0 & 0 & 0 \\
0 & 0 & \sigma_{\dot{\gamma}}^{2} & 0 & 0 & 0 & 0 \\
0 & 0 & 0 & \sigma_{R}^{2} & 0 & 0 & 0 \\
0 & 0 & 0 & 0 & \sigma_{\alpha}^{2} & 0 & 0 \\
0 & 0 & 0 & 0 & 0 & \sigma_{\beta}^{2} & 0 \\
0 & 0 & 0 & 0 & 0 & 0 & \sigma_{s f}^{2}
\end{array}\right] .
$$

The noise strengths, $\sigma_{i}$, were determined experimentally, and reflect the true noise variances of the signals involved.

$\nabla \mathrm{f}_{\mathrm{x}}(k)$ and $\nabla \mathrm{f}_{\mathrm{w}}(k)$ are given by the following:

$$
\nabla \mathbf{f}_{\mathbf{x}}(k)=\left[\begin{array}{ccccccc}
1 & 0 & f_{13} & f_{14} & f_{15} & 0 & 0 \\
0 & 1 & f_{23} & f_{24} & f_{25} & 0 & 0 \\
0 & 0 & 1 & f_{34} & f_{35} & f_{36} & 0 \\
0 & 0 & 0 & 1 & 0 & 0 & 0 \\
0 & 0 & 0 & 0 & 1 & 0 & 0 \\
0 & 0 & 0 & 0 & 0 & 1 & 0 \\
0 & 0 & 0 & 0 & 0 & 0 & 1
\end{array}\right]
$$

where

$$
\nabla \mathrm{f}_{\mathrm{w}}(k)=\left[\begin{array}{ccccccc}
g_{11} & 0 & 0 & 0 & 0 & 0 & 0 \\
g_{21} & 0 & 0 & 0 & 0 & 0 & 0 \\
g_{31} & g_{32} & g_{33} & 0 & 0 & 0 & 0 \\
0 & 0 & 0 & \Delta T & 0 & 0 & 0 \\
0 & 0 & 0 & 0 & \Delta T & 0 & 0 \\
0 & 0 & 0 & 0 & 0 & \Delta T & 0 \\
0 & 0 & 0 & 0 & 0 & 0 & \Delta T
\end{array}\right]
$$

where we first have the first set of equations shown at the bottom of the next page, and then we have the second set of equations following them.

\section{Observation Prediction}

The predicted observation is found by taking expectations conditioned on all previous observations, truncating at first order to give

$$
\begin{aligned}
\hat{\mathbf{z}}(k+1 \mid k) & =E\left[\mathbf{z}(k+1) \mid \mathbf{Z}^{k}\right] \\
& =\mathbf{h}(\hat{\mathbf{x}}(k+1 \mid k)) .
\end{aligned}
$$

If there is a predicted state for the vehicle, $\hat{\mathrm{x}}(k+1 \mid k)$, we can therefore predict the observations that will be made at that state. From (7) and (8) and from (18), we have the predicted

$$
\begin{aligned}
x(k+1) & =x(k)+\Delta T \omega(k) R(k) \cos (\alpha(k)+\phi(k)) \\
y(k+1) & =y(k)+\Delta T \omega(k) R(k) \sin (\alpha(k)+\phi(k)) \\
\phi(k+1) & =\phi(k)+\Delta T \frac{(\omega(k) R(k) \sin (\beta(k)-\alpha(k)+\gamma(k))-\dot{\gamma}(k) L \cos (\beta(k)))}{L(\cos (\beta(k))+\cos \beta(k)+\gamma(k)))} \\
R(k+1) & =R(k)+\Delta T \delta R(k) \\
\alpha(k+1) & =\alpha(k)+\Delta T \delta \alpha(k) \\
\beta(k+1) & =\beta(k)+\Delta T \delta \beta(k) \\
x_{s f}(k+1) & =x_{s f}(k)+\Delta T \delta \mathbf{w}_{s f}(k)
\end{aligned}
$$


observations as

$$
\begin{aligned}
\mathbf{z}(k+1 \mid k) & =\left[\begin{array}{c}
\hat{\mathbf{z}}_{\theta}^{i}(k+1 \mid k) \\
\hat{\mathbf{z}}_{\mathrm{gyro}}(k+1 \mid k)
\end{array}\right] \\
& =\left[\begin{array}{l}
\arctan \left(\frac{Y_{i}-\hat{y}(k+1 \mid k)}{X_{i}-\hat{x}(k+1 \mid k)}\right)-\hat{\phi}(k+1 \mid k) \\
\hat{\phi}(k+1 \mid k)+\hat{x}_{s f}(k+1 \mid k)
\end{array}\right] .
\end{aligned}
$$

Now, the innovation or observation prediction error covariance $\mathbf{S}(k)$, used in the calculation of the Kalman gains must be computed. This is found by squaring the estimated observation error and taking expectations of the first $k$ measurements to give the following:

$$
\begin{aligned}
\mathbf{S}(k+1)= & \nabla \mathbf{h}_{x}(k+1) \mathbf{P}(k+1 \mid k) \nabla \mathbf{h}_{x}^{T}(k+1) \\
& +\mathbf{R}(k+1) .
\end{aligned}
$$

In this case, the Jacobian $\nabla \mathbf{h}_{x}(k+1)$ is given by

$$
\begin{aligned}
& \nabla \mathbf{h}_{x}(k+1) \\
& \quad=\left[\begin{array}{ccccccc}
-\frac{\hat{y}(k+1 \mid k)-Y_{i}}{d^{2}} & \frac{\hat{x}(k+1 \mid k)-X_{i}}{d^{2}} & -1 & 0 & 0 & 0 & 0 \\
0 & 0 & 1 & 0 & 0 & 0 & 1
\end{array}\right]
\end{aligned}
$$

where $d=\sqrt{\left(X_{i}-\hat{x}(k+1 \mid k)\right)^{2}+\left(Y_{i}-\hat{y}(k+1 \mid k)\right)^{2}}$ is the predicted distance between a beacon and the vehicle.

The observation variance term $\mathbf{R}(\mathbf{k})$ is given by

$$
\mathbf{R}(k)=\left[\begin{array}{cc}
\sigma_{\theta}^{2} & 0 \\
0 & \sigma_{\text {gyro }}^{2}
\end{array}\right] \text {. }
$$

Again, the noise strengths, $\sigma_{\theta}$ and $\sigma_{\text {gyro, }}$, were determined experimentally to reflect the true noise variances of the signals involved.

\section{Update Equations}

The estimate (or update) of state and covariance is given by the following standard Kalman filter equations:

$$
\begin{aligned}
\hat{\mathbf{x}}(k+1 \mid k+1)= & \hat{\mathbf{x}}(k+1 \mid k)+\mathbf{W}(k+1)[\mathbf{z}(k+1) \\
& -\mathbf{h}(\hat{\mathbf{x}}(k+1 \mid k))] \\
\mathbf{P}(k+1) \mid k+1)= & \mathbf{P}(k+1) \mid k) \\
& -\mathbf{W}(k+1) \mathbf{S}(k+1) \mathbf{W}^{T}(k+1)
\end{aligned}
$$

where

$$
\mathbf{W}(k+1)=\mathbf{P}(k+1) \mid k) \nabla \mathbf{h}_{x}^{T}(k+1) \mathbf{S}(k+1)^{-1} .
$$

$$
\begin{aligned}
& f_{13}=-\Delta T \bar{\omega}(k) \hat{R}(k \mid k) \sin (\hat{\alpha}(k \mid k)+\hat{\phi}(k \mid k)) \\
& f_{14}=\Delta T \bar{\omega}(k) \cos (\hat{\alpha}(k \mid k)+\hat{\phi}(k \mid k)) \\
& f_{15}=-\Delta T \bar{\omega}(k) \hat{R}(k \mid k) \sin (\hat{\alpha}(k \mid k)+\hat{\phi}(k \mid k)) \\
& f_{23}=\Delta T \bar{\omega}(k) \hat{R}(k \mid k) \cos (\hat{\alpha}(k \mid k)+\hat{\phi}(k \mid k)) \\
& f_{24}=\Delta T \bar{\omega}(k) \sin (\hat{\alpha}(k \mid k)+\hat{\phi}(k \mid k)) \\
& f_{25}=\Delta T \bar{\omega}(k) \hat{R}(k \mid k) \cos (\hat{\alpha}(k \mid k)+\hat{\phi}(k \mid k)) \\
& f_{34}=\frac{\Delta T \bar{\omega}(k) \sin (\hat{\beta}(k \mid k)-\hat{\alpha}(k \mid k)+\bar{\gamma}(k))}{L(\cos (\hat{\beta}(k \mid k))+\cos (\hat{\beta}(k \mid k)+\bar{\gamma}(k)))} \\
& f_{35}=-\frac{\Delta T \bar{\omega}(k) \hat{R}(k \mid k) \sin (\hat{\beta}(k \mid k)-\hat{\alpha}(k \mid k)+\bar{\gamma}(k))}{L(\cos (\hat{\beta}(k \mid k))+\cos (\hat{\beta}(k \mid k)+\bar{\gamma}(k)))}
\end{aligned}
$$

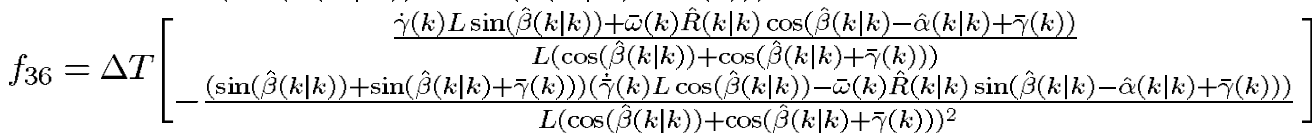

$$
\begin{aligned}
g_{11} & =\Delta T \hat{R}(k \mid k) \cos (\hat{\alpha}(k \mid k)+\hat{\phi}(k \mid k)) \\
g_{21} & =\Delta T \hat{R}(k \mid k) \sin (\hat{\alpha}(k \mid k)+\hat{\phi}(k \mid k)) \\
g_{31} & =\frac{\Delta T \hat{R}(k \mid k) \sin (\hat{\beta}(k \mid k)-\hat{\alpha}(k \mid k)+\bar{\gamma}(k))}{L(\cos (\hat{\beta}(k \mid k))+\cos (\hat{\beta}(k \mid k)+\bar{\gamma}(k)))} \\
g_{32} & =\Delta T\left[\begin{array}{c}
\frac{\bar{\omega}(k) \hat{R}(k \mid k) \cos (\hat{\beta}(k \mid k)-\hat{\alpha}(k \mid k)+\bar{\gamma}(k))}{L(\cos (\hat{\beta}(k \mid k))+\cos (\hat{\beta}(k \mid k)+\bar{\gamma}(k)))} \\
-\frac{\sin (\hat{\beta}(k \mid k)+\bar{\gamma}(k)))(\hat{\bar{\gamma}}(k) L \cos (\hat{\beta}(k \mid k))-\bar{\omega}(k) \hat{R}(k \mid k) \sin (\hat{\beta}(k \mid k)-\hat{\alpha}(k \mid k)+\bar{\gamma}(k)))}{L(\cos (\hat{\beta}(k \mid k))+\cos (\hat{\beta}(k \mid k)+\bar{\gamma}(k)))^{2}}
\end{array}\right] \\
g_{33} & =-\frac{\Delta T \cos (\hat{\beta}(k \mid k))}{\cos (\hat{\beta}(k \mid k))+\cos (\hat{\beta}(k \mid k)+\bar{\gamma}(k))}
\end{aligned}
$$




\section{REFERENCES}

[1] F. Labonte and P. Cohen, "Perceptual aspects of mining equipment teleoperation," in Proc. 6th Canadian Symp. Mining Automat., 1994.

[2] F. Labonte, J. Giraud, and V. Polotski, "Telerobotics issues in the operation of a LHD vehicle," in Proc. 3rd Canadian Conf. Comput. Appl. Mineral Ind., 1995.

[3] G. Baiden, "Multiple LHD teleoperation and guidance at Inco limited," in Proc. Int. Mining Congr., 1993.

[4] R. Herteau, M. St-Amant, Y. Laperriere, and G. Chevrette, "Optical guidance system for underground mine vehicles," in IEEE Int. Conf. Robot. Automat., 1992, pp. 639-644.

[5] K. Amdahl and M. Ludstrom, "Automatic truck saves money underground," World Mining, pp. 40-44, Nov. 1972.

[6] J. Steele, C. Ganesh, and A. Kleve, "Control and scale model simulation of sensor-guided LHD mining machines," IEEE Trans. Ind. Applicat. vol. 29, pp. 1232-1238, 1993.

[7] H. Makela, H. Lehtinen, K. Rintanen, and K. Koskinen, "Navigation system for LHD machines," Intell. Auton. Veh., pp. 314-319, 1995.

[8] N. Vagenas, M. Scoble, and G. Baiden, "A review of the first 25 years of mobile machine automation in underground hard rock mines," CIM Bull., vol. 90, no. 1006, pp. 57-62, Jan. 1997.

[9] H. F. Durrant-Whyte, "An autonomous guided vehicle for cargo handling applications," Int. J. Robot. Res., vol. 15, 1996.

[10] P. Maybeck, Stochastic Models, Estimation, and Control New York: Academic, 1979, vol. 1.

[11] A. Kelly, "A 3-D state space formulation of a navigation Kalman filter for autonomous vehicles," Tech. Rep., CMU Robot. Inst., 1994.

[12] R. Chatila and S. Lacroix, "Adaptive navigation for autonomous mobile robots," in Proc. Int. Symp. Robot. Res., 1995.

[13] B. Barshan and H. F. Durrant-Whyte, "Inertial navigation systems for mobile robots," IEEE Trans. Robot. Automat., vol. 11, pp. 328-342, 1995.

[14] S. Sukkarieh, E. Nebot, and H. Durrant-Whyte, "The GPS aiding of INS for land vehicle navigation," in Proc. Int. Conf. Field Service Robot., 1997.

[15] , "Achieving integrity in an INS-GPS navigation loop for autonomous land vehicle applications," in Proc. IEEE Int. Conf. Robot. Automat., 1998.

[16] S. Scheding, E. M. Nebot, M. Stevens, H. F. Durrant-Whyte, J. Roberts, P. Corke, J. Cunningham, and B. Cook, "Experiments in autonomous underground guidance," in Proc. IEEE Conf. Robot. Automat., 1997.

Steven Scheding received the Ph.D. degree in mechatronics from the University of Sydney, Australia, in 1998.

$\mathrm{He}$ is a Postdoctoral Researcher with the National Robotics Engineering Consortium, Carnegie Mellon University, Pittsburgh, PA. Research interests include the automation of large outdoor vehicles, particularly in mining, fault detection and identification, simultaneous localization and map building, nonlinear control, and system design.
Gamini Dissanayake received the degree in mechanical and production engineering from the University of Peradeniys, Sri Lanka and the M.Sc. degree in machine tool technology and the Ph.D. degree in mechanical engineering from the University of Birmingham, U.K., in 1981 and 1985, respectively.

$\mathrm{He}$ is a Senior Lecturer in Mechanical and Mechatronic Engineering at the University of Sydney, Australia. His research interests are in dynamics and control of mechanical systems, mobile robots, navigation systems, optimization, and path planning.

Dr. Dissanayake is a member of the Australian Centre for Field Robotics where he is involved in a number of automation projects in mining and stevedoring.

Eduardo Mario Nebot received the B.S. degree in electrical engineering from the Universidad Nacional del Sur, Argentina and the M.S. and Ph.D. degrees from Colorado State University, Fort Collins.

He is a Senior Lecturer with the Department of Mechanical and Mechatronic Engineering, University of Sydney. He has been a faculty member since 1992. His current research consists of inertial sensors, laser ranging, GPS and inertial sensing in land vehicle applications, navigation and guidance algorithms, decentralized and distributed navigation, fault detection, sensorbased map-building, and terrain-aiding. He has also been involved in different automation projects in the stevedoring, mining, and cargo handling industries.

Dr. Nebot has been a member of the Institute of Electrical and Electronic Engineering since 1978 .

Hugh Durrant-Whyte received the B.Sc. degree in nuclear and mechanical engineering from the University of London, U.K. in 1983, and the M.S.E. and Ph.D. degrees from the University of Pennsylvania, Philadelphia, in 1985 and 1986, respectively.

From 1986 to 1995, he was a University Lecturer in Engineering Science with the University of Oxford, U.K. Since 1995, he has been Professor of Mechatronics at the University of Sydney, Australia. His current research interests include data fusion and robotics. 